\title{
Comprehensive cardiac resynchronization therapy optimization in the real world
}

\author{
Jan Steffel ${ }^{1}$, Helene Rempel ${ }^{1}$, Alexander Breitenstein ${ }^{1}$, Susann Schmidt ${ }^{1}$, \\ Mehdi Namdar ${ }^{2}$, Nazmi Krasniqi ${ }^{1}$, Johannes Holzmeister ${ }^{1}$, Thomas F. Lüscher ${ }^{1}$, \\ Frank Ruschitzka ${ }^{1}$, David Hürlimann ${ }^{1}$ \\ ${ }^{1}$ Department of Cardiology, Cardiovascular Center, University Hospital Zurich, Switzerland \\ ${ }^{2}$ Heart Rhythm Management Centre, UZB, Brussels, Belgium
}

\begin{abstract}
Background: Cardiac resynchronization therapy (CRT) reduces morbidity and mortality in patients suffering from chronic heart failure (CHF). Optimal device programming is crucial for maximum patient benefit. The goal of the present study was to assess device settings from CHF patients undergoing CRT optimization in a "real world" setting, and to delineate parameters most frequently requiring adjustment.

Methods: All patients who underwent CRT device implantation in the Cardiology Clinic at the University Hospital Zurich between January 2011 and September 2012 and in whom follow-up was available were included in this analysis.

Results: A total of 170 CHF patients were included in this analysis. True biventricular pacing was present in $44 \%$ of all patients, while QRS fusion was detected in $49.9 \%$. The majority of the patients presented with suboptimal atrioventricular $(A V)$ delays requiring adjustment. $A V$ delays were therefore shortened due to the presence of QRS fusion in $53.3 \%$ and $38.1 \%$ of patients ( $A V$ and $p A V$, respectively) or prolonged because of truncation of the $A$ wave in the left ventricular inflow pulse wave Doppler measurement (17.5\% and $28.4 \%$ for sAV and $p A V$, respectively). In contrast, interventricular delay (VV delay) was rarely changed (11.9\%).

Conclusions: In our "real world" cohort, a substantial proportion of patients presented to their first post-operative consultation with suboptimal device settings. Our data indicate that the opportunity to optimize device settings is frequently wasted in the "real world", underlining the necessity for expert device follow-up to deliver optimal care to this challenging group of heart failure patients. (Cardiol J 2014; 21, 3: 316-324)
\end{abstract}

Key words: heart failure, cardiac resynchronization therapy, integrative device follow-up

\section{Introduction}

Cardiac resynchronization therapy (CRT) has become a cornerstone in the treatment of chronic heart failure (CHF) $[1,2]$. Indeed, several clinical trials observed a reduction in morbidity and mortality as compared to medical therapy alone in patients suffering from symptomatic left ventricular (LV) dysfunction (LV ejection fraction [LVEF] $\leq 35 \%$ ) with a prolonged $\mathrm{QRS}$ duration $(\geq 120 \mathrm{~ms})$.

Address for correspondence: Jan Steffel, MD, FESC, Co-chair Cardiac Arrhythmia Division, Department of Cardiology, University Hospital Zurich, Raemistrasse 100, CH-8091 Zurich, Switzerland, tel: +41 4425511 11, fax: +41 442558701 , e-mail: j.steffel@gmx.ch

Received: 12.06.2013 Accepted: 13.08.2013 
Nevertheless, almost $30 \%$ of patients do not exhibit an improvement in clinical symptoms or hemodynamic parameters after CRT implantation (so-called "non-responders") [3]. This lack of improvement may be due to pre-implantation characteristics such as large areas of scar tissue due to coronary artery disease or a lack of mechanical dyssynchrony [4]. Suboptimal LV lead position further represents a reason for a lack of benefit [4]. Additionally, however, some patients do not respond to CRT due to suboptimal CRT device settings, impairing LV filling (atrioventricular [AV] dyssynchrony) and/or persistent LV dyssynchrony [5].

At the University Hospital of Zurich, we implemented a standard protocol by which every patient with a CRT device undergoes a complete device optimization 3-6 months after implantation and again on a yearly basis or if clinically deemed necessary. The goal of the present study was to summarize clinical presentation, echocardiographic findings, and device settings from CHF patients undergoing CRT optimization in this "real world" setting, unraveling the amount of patients presenting with suboptimal settings, as well as the parameters most frequently requiring adjustment. We provide a rationale for the necessity of implementing a routine protocol for the integrated management of these complex CHF patients, including expert device management.

\section{Methods}

\section{Study population and CRT implantation}

All patients with a CRT device receiving their first device follow-up in our specialized device clinic from January $1^{\text {st }} 2011$ until September 2012 were prospectively included. The study was approved by the cantonal ethics committee Zurich. Mean time from implantation to optimization was 2.9 months (Table 1 ). Indications for CRT implantation were based on current guidelines [1]. CRT devices were implanted by a standard procedure under local anesthesia. Devices and leads from Biotronik, Boston Scientific/Guidant, Medtronic and St. Jude Medical were used. For implantation of the LV lead, percutaneous placement into a lateral or postero-lateral vein was attempted whenever possible. If no suitable vein branch was available, epicardial lead implantation was performed.

\section{Follow-up protocol}

We implemented a standard protocol by which every patient with a CRT device underwent a comprehensive and standardized device follow-up
Table 1. Demographics at implantation.

\begin{tabular}{lc}
\hline Men [years] & $130 / 170(76.5 \%)$ \\
New implantation & $129 / 170(75.9 \%)$ \\
CRT upgrade & $41 / 170(24.1 \%)$ \\
Time implantation & $2.9 \pm 5$ \\
- optimization [months] & $62.8 \pm 12.5$ \\
Age at implantation [years] & $75 / 169(44.4 \%)$ \\
Ischemic cardiomyopathy & $156 / 170(91.8 \%)$ \\
CRT-D & $107 / 167(64.1 \%)$ \\
Sinus rhythm at implantation & \\
\hline
\end{tabular}

CRT — cardiac resynchronization therapy

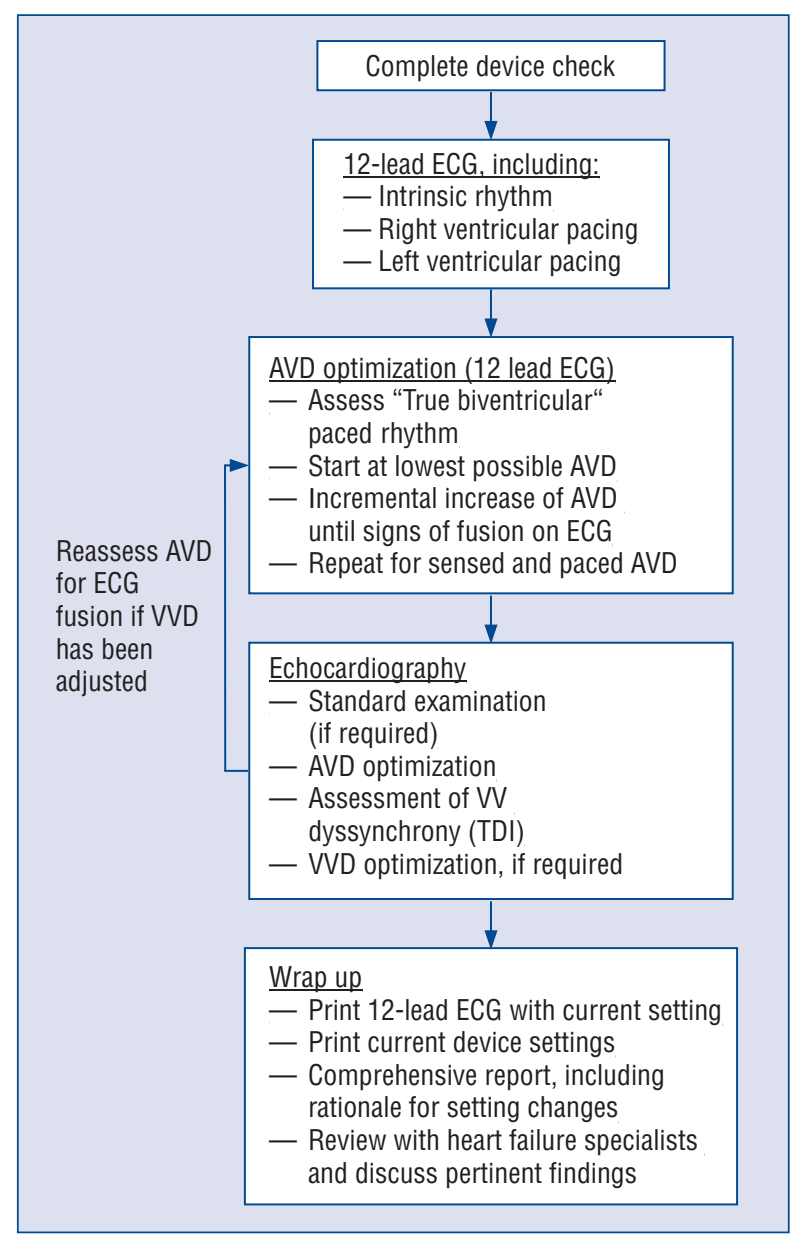

Figure 1. Cardiac resynchronization therapy patient follow-up algorithm as implemented at the University of Zurich. See text for details, AVD - atrioventricular delay; VVD - interventricular delay.

and optimization procedure (Fig. 1). After a complete clinical assessment by a heart failure specialist, a comprehensive device follow-up was performed 
by implantable electronic cardiac device specialists (JS/DH), including complete check of the system with 12-lead electrocardiogram (ECG), and review of all brady- and tachycardia parameters. Subsequently, a complete echocardiographic exam was performed (Vivid E9, GE), followed by optimization of the AV- and interventricular (VV) delay if necessary. AV delay optimization was performed starting at a long AV-delay without intrinsic conduction. The AV-delay was then progressively shortened in steps of $20 \mathrm{~ms}$ until truncation of the A-wave was observed. Then, the AV-delay was prolonged in $10 \mathrm{~ms}$ steps until the optimal separation of $\mathrm{E} / \mathrm{A}$ wave without truncation of the A-wave was reached, which was considered the optimal AV-delay.

\section{Results}

\section{Study population}

A total of $170 \mathrm{CHF}$ patients were included in this prospective analysis. Baseline demographic data at implantation, as well as clinical characteristics at time of follow-up are summarized in Table 1. The majority of patients were men $(76.5 \%)$, and had a cardiovascular risk profile typical of a real-world CHF cohort as previously shown [6]. All patients were on optimal medical therapy including angiotensin converting enzyme-inhibitors or angiotensin-II-blockers (95.2\%), beta-blockers (93.5\%) and diuretics (86.3\%) prior to implantation. Furthermore, more than $50 \%$ of patients were treated with an aldosterone antagonist (55.4\%) in addition to standard diuretic therapy, and received anti-arrhythmic treatment with either digitalis $(13.1 \%)$ or amiodarone $(17.9 \%)$ (Table 2$)$.

\section{Echocardiographic and electrocardiographic parameters at follow-up}

Most patients demonstrated a reduced LVEF (mean $37.3 \pm 11.1 \%$ ) and a dilated LV (end-diastolic volume index $88.4 \pm 41.6 \mathrm{~mL} / \mathrm{m}^{2}$; Table 3 ). Of note, parameters of dyssynchrony at this time mainly demonstrated values within our lab's predefined normal values (interventricular mechanical delay - IVMD $<40 \mathrm{~ms}$, tissue Doppler imaging $-\mathrm{TDI}_{\text {septal-lateral }}<60 \mathrm{~ms}, \mathrm{TDI}_{\text {anteroseptal-posterolateral }}$ $<60 \mathrm{~ms}$ ), with an IVMD of $17.5 \pm 27.7$, as well as $32.7 \pm 46.8 \mathrm{~ms}$ and $33.5 \pm 46.3 \mathrm{~ms}$ for septal-lateral and anteroseptal-posterolateral delay assessed by TDI, respectively. The mean intrinsic QRS duration was $150 \pm 26.6 \mathrm{~ms}$ with an ECG pattern of left bundle branch block in the majority of cases (59.5\%), and an average PQ interval of $184.7 \pm$
Table 2. Clinical parameters at time of optimization.

\begin{tabular}{lc}
\hline Clinical parameters & $1.7 \pm 0.1$ \\
Height $[\mathrm{m}]$ & $82 \pm 18.5$ \\
Weight $[\mathrm{kg}]$ & $28.4 \pm 5.9$ \\
Body mass index $\left[\mathrm{kg} / \mathrm{m}^{2}\right]$ & $116.1 \pm 17.3$ \\
Systolic BP $[\mathrm{mm} \mathrm{Hg}]$ & $73 \pm 11.7$ \\
Diastolic BP $[\mathrm{mm} \mathrm{Hg}]$ & \\
NYHA class: & $24 / 134(17.9 \%)$ \\
NYHA I & $54 / 134(40.3 \%)$ \\
NYHA II & $20 / 134(14.9 \%)$ \\
NYHA III & $1 / 134(0.7 \%)$ \\
NYHA IV & \\
Medication & $157 / 168(93.5 \%)$ \\
Beta-blocker & $160 / 168(95.2 \%)$ \\
ACE-I/ARB & $93 / 168(55.4 \%)$ \\
Aldosterone antagonist & $145 / 168(86.3 \%)$ \\
Other diuretics & $22 / 168(13.1 \%)$ \\
Digitalis & $30 / 168(17.9 \%)$ \\
Amiodarone & $11 / 168(6.5 \%)$ \\
CCB & $11 / 168(6.5 \%)$ \\
Nitrates & $103 / 168(61.3 \%)$ \\
Lipid lowering treatment & $84 / 168(50 \%)$ \\
Aspirin & $12 / 168(7.1 \%)$ \\
Clopidogrel & $84 / 168(50 \%)$ \\
Oral anticoagulation & \\
\hline ACE-IARB ang
\end{tabular}

ACE-I/ARB - angiotensin-converting-enzyme inhibitors/angiotensin II receptor blockers; $\mathrm{BP}$ - blood pressure; CCB — calcium channel blocker; NYHA — New York Heart Association classification

$\pm 27.6 \mathrm{~ms}$ (Table 4 ). At the time of implantation and at the first follow-up, most patients were in sinus rhythm $(65.9 \%)$. While $65.3 \%$ of the patients had the same rhythm at our follow-up visit, a minority of patients switched from atrial fibrillation to sinus rhythm $(2.4 \%)$ and vice versa $(4.8 \%)$.

\section{Device settings and intervals at the time of optimization}

True biventricular pacing was present in nearly half of the patients at the time of optimization (44.7\%), while QRS fusion could be detected in $39.4 \%$ of cases (Table 5, Fig. 2). At the time of optimization, average sensed AV (sAV) delay was $110.5 \pm 19 \mathrm{~ms}$ and paced AV (pAV) $136.5 \pm 23.4 \mathrm{~ms}$. QRS fusion, as diagnosed by 12-lead surface ECG, began to appear at an average sAV of $103.9 \pm$ $\pm 37.1 \mathrm{~ms}$ and an average pAV of $136.5 \pm 23.4 \mathrm{~ms}$, respectively.

A biventricular pacing rate of more than $95 \%$ was present in $85.8 \%$ of patients from the studied cohort. The main reason for a low biventricular pacing percentage was either atrial fibrillation or 
Table 3. Echocardiography at optimization.

\begin{tabular}{|c|c|}
\hline LVEF [\%] & $37.3 \pm 11.1$ \\
\hline LVEDD [mm] & $6.2 \pm 1.1$ \\
\hline LVESD $[\mathrm{mm}]$ & $4.9 \pm 1.3$ \\
\hline $\mathrm{EDV}[\mathrm{mL}]$ & $169.5 \pm 79.2$ \\
\hline EDV index $\left[\mathrm{mL} / \mathrm{m}^{2}\right]$ & $88.4 \pm 41.6$ \\
\hline $\mathrm{ESV}[\mathrm{mL}]$ & $110.8 \pm 71.5$ \\
\hline ESV index $\left[\mathrm{mL} / \mathrm{m}^{2}\right]$ & $58.8 \pm 38.3$ \\
\hline \multicolumn{2}{|l|}{ Diastolic dysfunction: } \\
\hline No diastolic dysfunction & $92 / 168(54.8 \%)$ \\
\hline Grade I & $7 / 168(4.2 \%)$ \\
\hline Grade II & $1 / 168(0.6 \%)$ \\
\hline Grade III & $0 / 168(0 \%)$ \\
\hline \multicolumn{2}{|l|}{ Mitral regurgitation: } \\
\hline Minimal & $88 / 167(52.7 \%)$ \\
\hline Mild & $59 / 167(35.3 \%)$ \\
\hline Moderate & $13 / 167(7.8 \%)$ \\
\hline Severe & $4 / 167(2.4 \%)$ \\
\hline LAESD [mm] & $4.7 \pm 0.9$ \\
\hline RV: Area D $\left[\mathrm{cm}^{2}\right]$ & $16.2 \pm 5.1$ \\
\hline Fractional shortening RV [\%] & $39.7 \pm 12.1$ \\
\hline TAM [mm] & $18.3 \pm 4.9$ \\
\hline RV/RA-pressure gradient [mm Hg] & $27.9 \pm 11.6$ \\
\hline RA size & $5 \pm 0.9$ \\
\hline \multicolumn{2}{|l|}{ Dyssynchrony assessment: } \\
\hline RV-PEP [ms] & $118.1 \pm 31.3$ \\
\hline LV-PEP [ms] & $135.5 \pm 31.5$ \\
\hline IVMD & $17.5 \pm 27.7$ \\
\hline TDI SL & $32.7 \pm 46.8$ \\
\hline TDI AS-IL & $33.5 \pm 46.3$ \\
\hline Diastolic filling time/RR-interval & $49.5 \pm 32.1$ \\
\hline
\end{tabular}

AS-AL — anteroseptal-posterolateral; EDV - end-diastolic volume; ESV - end-systolic volume; IVMD - interventricular mechanical delay; LAESD - left atrium end-diastolic diameter; LV - left vendelay; LAESD - left atrium end-diastolic diameter; LV — left ven-
tricle; LVEF — left ventricular ejection fraction; LVEDD - left ventricular end-diastolic diameter; LVESD - left ventricular end-diastolic diameter; PEP — preejection period; RV — right ventricle; RA right atrium; SL - septal lateral; TDI - tissue Doppler imaging; TAM — tricuspid annular movement

frequent ventricular extrasystoles. At the time of follow-up, mean programmed VV delay was $8.5 \pm 14.4 \mathrm{~ms}$ (LV first).

\section{Left ventricular lead settings}

In a third of all cases, lead pacing configuration was "true" bipolar from the LV lead tip (LvTip) to the LV ring (LvRing; $34.1 \%$ ) or between LvTip to the right ventricular lead ring (RvRing; $28.2 \%$, Table 6). In every fifth patient, the pacing vector was between the LvTip and the coil of the right ventricular lead (RvCoil; 23.5\%). With these configurations, only a minority of patients $(\mathrm{n}=7,4.2 \%)$ were suffering from diaphragmatic capture $(4.2 \%)$, which was solved in all cases by reprogramming.
Table 4. Electrocardiogram parameters.

\begin{tabular}{lc}
\hline Rhythm at optimization & \\
SR & $112 / 170(65.9 \%)$ \\
SR, AVB III ${ }^{\circ}$ without & $15 / 170(8.8 \%)$ \\
ventricular escape & \\
SR, AVB III ${ }^{\circ}$ with & $6 / 170(3.5 \%)$ \\
ventricular escape & \\
AF with AV conduction & $20 / 170(11.8 \%)$ \\
AF, AVB III & $7 / 170(4.1 \%)$ \\
AF post AV node ablation & $6 / 170(3.5 \%)$ \\
PQ intrinsic & $184.7 \pm 27.6$ \\
QRS intrinsic & $150 \pm 26.6$ \\
Bundle branch block: & \\
No block & $14 / 163(8.6 \%)$ \\
LBBB & $97 / 163(59.5 \%)$ \\
RBBB & $13 / 163(8 \%)$ \\
AVB III & $36 / 163(22.1 \%)$ \\
Development of rhythm & \\
Unchanged & $109(65.3 \%)$ \\
AF $\rightarrow$ SR & $4(2.4 \%)$ \\
AF $\rightarrow$ PM & $2(1.2 \%)$ \\
SR $\rightarrow$ AF & $8(4.8 \%)$ \\
SR $\rightarrow$ PM & $4(2.4 \%)$ \\
PM upgrade & $41(24 \%)$ \\
\hline
\end{tabular}

$\mathrm{AF}$ - atrial fibrillation; $\mathrm{AV}$ - atrioventricular; AVB - atrioventricular block; LBBB - left bundle branch block; RBBB — right bundle branch block; PM — pacemaker; SR — sinus rhythm

\section{Optimization of CRT settings after implantation}

In the majority of all patients who underwent CRT device implantation, the programmed AV intervals were deemed suboptimal during follow-up and were subsequently reprogrammed (Table 7 ). AV delays were shortened (sAV delay in $53.3 \%$, pAV delay in $38.1 \%$ of the patients) mainly due to the presence of QRS fusion on the 12-lead ECG. In contrast, the AV delay was prolonged in only $17.5 \%$ and $28.4 \%$ (sAV and pAV, respectively), mostly due to truncation of the A wave in the LV inflow pulse wave Doppler measurement. In 77 patients (45\% of the entire cohort), some degree of QRS fusion was accepted in order to allow for better LV filling.

In contrast to the adjustments of the AV interval, the VV delay was left unchanged in the majority of patients $(88.1 \%)$. The main reason for a change in VV delay was intraventricular dyssynchrony observed on TDI (82.4\%).

Thirty three (19.4\%) patients in our cohort suffered from atrial fibrillation. By virtue of this, AV optimization was impossible in these patients and VV optimization greatly impaired. Of our patients with atrial fibrillation, 20 (61\%) had intrinsic con- 
Table 5. AV/VV settings at optimization.

\begin{tabular}{|c|c|}
\hline \multicolumn{2}{|l|}{ Pacing at optimization: } \\
\hline True biventricular stimulation & $76 / 170(44.7 \%)$ \\
\hline Fusion & $67 / 170(39.4 \%)$ \\
\hline Pseudofusion & $3 / 170(1.8 \%)$ \\
\hline Atrial fibrillation & $23 / 170(13.5 \%)$ \\
\hline \multicolumn{2}{|l|}{ AV delays: } \\
\hline sAV (at interrogation) & $110.5 \pm 19$ \\
\hline pAV (at interrogation) & $136.5 \pm 23.4$ \\
\hline Begin QRS fusion (sAV) & $103.9 \pm 37.1$ \\
\hline Begin QRS fusion (pAV) & $174.3 \pm 42.7$ \\
\hline Intraatrial delay (pAV-sAV) & $71.9 \pm 28.2$ \\
\hline No fusion (AV block) & $33(19.4 \%)$ \\
\hline Dynamic AV delay on & $78 / 164(47.6 \%)$ \\
\hline \multicolumn{2}{|l|}{ Situation at sensed AV delay 120 ms: } \\
\hline Pure biventricular stimulation & $58 / 140(41.4 \%)$ \\
\hline Fusion & $72 / 140(51.4 \%)$ \\
\hline Intrinsic rhythm/Pseudofusion & $8 / 140(5.7 \%)$ \\
\hline \multicolumn{2}{|l|}{ Status before optimization: } \\
\hline A-wave truncation & $38 / 147(25.9 \%)$ \\
\hline QRS fusion & $39 / 147(26.5 \%)$ \\
\hline $\begin{array}{l}\text { Both A-wave truncation } \\
\text { and QRS fusion }\end{array}$ & $31 / 147(21.1 \%)$ \\
\hline VV (at interrogation) [ms] & $8.5 \pm 14.4$ \\
\hline Biventricular pacing (average) [\%]: & $94.3 \pm 13.3$ \\
\hline$\geq 97 \%$ & $112(67.9 \%)$ \\
\hline$<97 \%$ & $53(32.1 \%)$ \\
\hline$<95 \%$ & $40(24.2 \%)$ \\
\hline$<90 \%$ & $23(13.9 \%)$ \\
\hline$\leq 85 \%$ & $16(9.7 \%)$ \\
\hline \multicolumn{2}{|c|}{ Reason for low biventricular pacing $(<95 \%)$ : } \\
\hline Atrial fibrillation & $18 / 39(46.2 \%)$ \\
\hline VES & $13 / 39(33.3 \%)$ \\
\hline Intrinsic conduction & $3 / 39(7.7 \%)$ \\
\hline Other & $4 / 39(10.3 \%)$ \\
\hline \multicolumn{2}{|l|}{ Device manufacturer: } \\
\hline Medtronic & $31 / 170(18.2 \%)$ \\
\hline St. Jude Medical & $49 / 170(28.8 \%)$ \\
\hline Biotronik & $79 / 170(46.5 \%)$ \\
\hline Boston Scientific & $10 / 170(5.9 \%)$ \\
\hline
\end{tabular}

$\mathrm{AV}$ - atrioventricular; sAV — sensed $A V ; \mathrm{pAV}$ - paced $A V$; VV interventricular; VES - ventricular extrasystoles

duction, whereas $7(21.2 \%)$ and $6(18.2 \%)$ patients had no intrinsic AV conduction or had undergone AV node ablation, respectively (Table 4).

\section{Discussion}

The aim of the present study was to give an overview on CRT settings at the time of device implantation and to demonstrate the potential for optimization by comprehensive assessment during

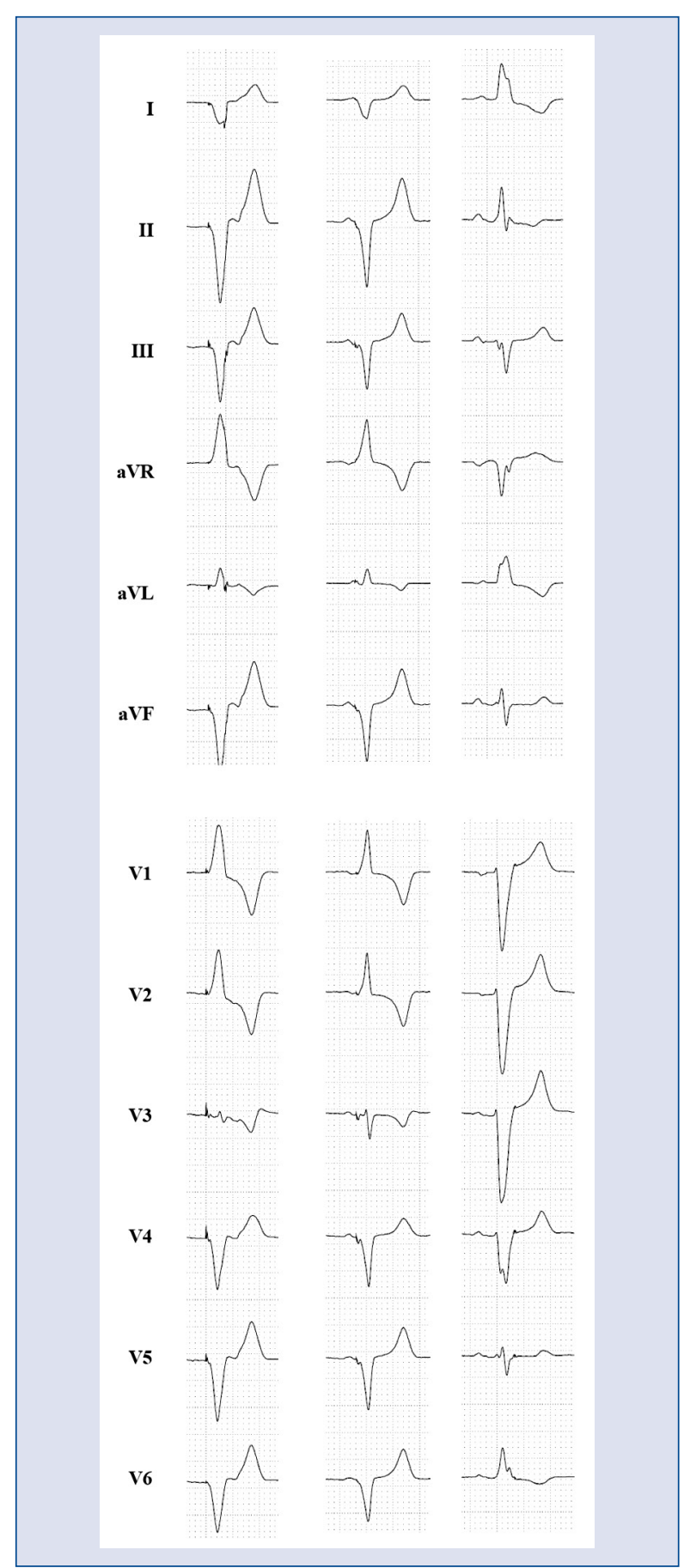

Figure 2. Twelve-lead electrocardiograms (ECGs) of a patient presenting for cardiac resynchronization therapy optimization. "True" biventricular pacing (VVI 90 bpm, left panel), intrinsic rhythm (right panel) and rhythm at follow-up (middle panel) are shown. Note the significant degree of QRS fusion as demonstrated by 12-lead ECG with the current device settings. ORS fusion is best appreciated in I, aVL and V3, indicating the necessity for comprehensive 12-lead ECG analysis in the follow-up of these patients. 
Table 6. Left ventricular lead parameters.

\begin{tabular}{lc}
\hline \multicolumn{2}{l}{ Left ventricular pacing configuration: } \\
LvTip-LvRing & $58 / 170(34.1 \%)$ \\
LvRing-LvTip (inverse bipolar) & $5 / 170(2.9 \%)$ \\
LvTip-RvRing & $48 / 170(28.2 \%)$ \\
LvRing-RvRing & $9 / 170(5.3 \%)$ \\
LvTip-RvCoil & $40 / 170(23.5 \%)$ \\
LvRing-RvCoil & $7 / 170(4.1 \%)$ \\
Unipolar & $1 / 170(0.6 \%)$ \\
Diaphragmatic capture: & \\
No diaphragmatic capture & $161 / 168(95.8 \%)$ \\
Diaphragmatic capture, & $7 / 168(4.2 \%)$ \\
resolved with reprogramming & \\
Diaphragmatic capture, not ame- & $0 / 168(0 \%)$ \\
nable to programming changes & \\
Threshold left ventricle [V] & $1.3 \pm 0.8$ \\
Threshold left ventricle [ms] & $0.5 \pm 0.2$ \\
Left ventricular sensing [mV] & $12.6 \pm 8.6$ \\
Left ventricular impedance [Ohm] & $644.4 \pm 228.3$ \\
\hline
\end{tabular}

Lv — left ventricle Rv — right ventricle

Table 7. Changes during optimization.

\begin{tabular}{lc}
\hline Change in sensed AV delay & \\
Unchanged & $39 / 137(28.5 \%)$ \\
Shortened & $73 / 137(53.3 \%)$ \\
Lengthened & $24 / 137(17.5 \%)$ \\
Changes in paced AV delay & \\
Unchanged & $45 / 134(33.6 \%)$ \\
Shortened & $51 / 134(38.1 \%)$ \\
Lengthened & $38 / 134(28.4 \%)$ \\
Reason for AV delay change & \\
ECG fusion & $56 / 97(57.7 \%)$ \\
LV inflow truncation & $23 / 97(23.7 \%)$ \\
LV inflow fusion & $14 / 97(14.4 \%)$ \\
Visual LV filling & $3 / 97(3.1 \%)$ \\
Other & $1 / 97(1 \%)$ \\
Status after optimization & \\
A-wave truncation & $22 / 147(15 \%)$ \\
QRS fusion & $47 / 147(32 \%)$ \\
Both A-wave truncation & $34 / 147(23.1 \%)$ \\
and QRS fusion & \\
Change in VV delay & \\
Unchanged & \\
LV earlier & $118 / 168(70.2 \%)$ \\
RV earlier & $13 / 168(7.7 \%)$ \\
Dyssynchronous, & $5 / 168(3 \%)$ \\
not improvable & $30 / 168(17.9 \%)$ \\
Reason for VV delay changes & \\
Visual & \\
Tissue Doppler imaging & $14 / 17(17.6 \%)$ \\
\hline & \\
\hline
\end{tabular}

$\mathrm{AV}$ - atrioventricular; ECG - electrocardiogram; LV — left ventricle; RV — right ventricle; VV — interventricular follow-up in an integrative device clinic. In view of the substantial discrepancy between programmed parameters and optimal values, our data indicate that this opportunity is frequently missed in the "real world", providing a rationale for the necessity of protocol-oriented expert follow-up and optimization procedures for these patients.

\section{AV delay optimization and QRS fusion}

Whether and how AV intervals should regularly be evaluated and adjusted remains a matter of debate [7-9]. In various preliminary studies, optimization of the AV delay has been demonstrated to significantly increase hemodynamic response, New York Heart Association (NYHA) class, LVEF and B-type natriuretic peptide level in the short-time follow-up [10-14]. In contrast, the recently published SmartDelay determined AV Optimization: A comparison to Other AV Delay Methods Used in Cardiac Resynchronization Therapy (SMART-AV) trial implied otherwise. In this study, CRT device recipients were randomized to a fixed empirical AV delay of $120 \mathrm{~ms}$, an echocardiographically optimized AV delay or a device-based AV optimization algorithm (SmartDelay) [15]. The primary endpoint, LV end-systolic volume at 6 months after implantation did not differ between the 3 groups. As discussed by the authors, it may be possible that the observed acute beneficial hemodynamic effects after CRT implantation are not sufficient enough to result in an improvement of hard clinical endpoints. On the other hand, the follow-up period of only 6 months may have been too short to evaluate such endpoints.

Furthermore, it has to be kept in mind that the optimal AV delay may have a high variability among CRT patients $[5,16]$. As such, data from SMART-AV do not imply that individual patients with suboptimal AV delay may not profit from an optimization procedure. Indeed, this has been substantiated most recently in a subanalysis from MADIT-CRT, in which patients with short AV-delays (notably $<120 \mathrm{~ms}$ ) had a superior outcome compared to those with longer AV delays [17]. Our data demonstrate that programming an empirical setting of $120 \mathrm{~ms}$ for the sensed AV delay was suboptimal in terms of true biventricular stimulation in the vast majority of patients $(57.1 \%)$, and $8(5.7 \%)$ patients even demonstrated entirely intrinsic conduction or pseudofusion (and hence loss of biventricular pacing) at this setting. Furthermore, a subanalysis of the Clinical Evaluation on Advanced Resynchronization (CLEAR) pilot study revealed that systematic CRT optimization was 
associated with a higher percentage of improved patients based on the composite endpoint (all-cause mortality, heart failure-related hospitalization, NYHA functional class, and quality of life score), fewer deaths and fewer hospitalizations [18]. These data clearly indicate a role for CRT optimization over standard programming in all patients.

Moreover, the mean interatrial delay was markedly longer $(71.9 \pm 28.2 \mathrm{~ms}$; Table 5$)$ than the standard programmed difference between sensed and paced AV delay (usually $30-40 \mathrm{~ms}$ ) found in the default settings, further supporting an individualized approach to AV delay programming.

The majority of our patients $(>70 \%)$ underwent reprogramming of the AV delay. One of the most important parameters to guide AV delay optimization is the level of true biventricular pacing or, vice versa, the degree of QRS fusion as a result of intrinsically conducted ventricular excitation [19-21]. In our cohort, $45.3 \%$ of patients presented with an AV delay too long to avoid any QRS fusion. However, whether some degree of ventricular fusion may be clinically beneficial remains a matter of debate, as a certain amount of QRS fusion has been shown to improve hemodynamics [22-24]. Fusion allows for intrinsic excitation of the right ventricle via the normal-conducting right bundle branch which may result in improved right ventricle contraction [25]. A recent study further demonstrated that the maximal rate of $L V$ pressure increase $(\mathrm{dP} / \mathrm{dt})$ was higher in LV pacing combined with intrinsic conduction as compared to biventricular pacing [22]. Based on these data, we accepted some degree of QRS fusion in order to allow for optimal LV filling in those cases where it was impossible to shorten the AV delay to the point of complete loss of QRS fusion (Fig. 2). Importantly, intrinsic conduction and pseudofusion could entirely and sustainably be avoided in all patients presenting with these findings.

\section{VV delay optimization}

For various reasons, VV delay optimization appears to be less important as compared to AV delay optimization. Even though an improvement in hemodynamic conditions has been observed after optimization of VV intervals [26-28], other randomized trials failed to find a beneficial effect $[29,30]$. The latter may, at least in part, be due to the fact that VV delay optimization was performed in the majority of patients on top of AV delay optimization. In a small study investigating the effect of simultaneous $\mathrm{AV}$ and VV time optimization, an additional but smaller beneficial effect of VV delay optimization was found [31]. In our cohort, only a minority of patients underwent VV optimization, mainly due to the fact that it was not deemed necessary due to satisfying echocardiographic dyssynchrony parameters and, coherently, visual impression of synchronous LV contraction. In patients who were optimized, the indication was mainly driven by TDI values during echocardiographic evaluation. Conversely, 30 (17.9\%) patients were dyssynchronous either visually or by TDI measurements, but could not be corrected by advancing left or right ventricle activation. As a result, and due to the lack of clear evidence for a benefit, VV optimization is only performed in special cases in our institution.

\section{CRT in patients with atrial fibrillation}

$19.4 \%$ of patients in our cohort suffered from atrial fibrillation. These patients pose a challenge in $\mathrm{CRT}$ as the uncoordinated and often rapid intrinsic conduction often results in a substantially impaired rate of biventricular pacing $(<95 \%)$. Indeed, atrial fibrillation was the main reason for a low percentage of biventricular pacing in our cohort. Pharmacologically, amiodarone has been shown to be most effective and safe for rhythm control in atrial fibrillation patients with heart failure [32], and, as a consequence, is frequently used to increase the percentage of biventricular stimulation. If the medical therapy is insufficient, AV nodal ablation is recommended as the next step [33]. In our cohort, $39.4 \%$ of patients with atrial fibrillation ultimately had no intrinsic AV conduction and, consequently, had a high degree of biventricular pacing.

\section{Limitations of the study}

Our study has some inherent limitations. Data are only collected from a single tertiary care center, and may hence not be generalizable to other healthcare settings. We do, however, believe that most interpretations and statements characteristically reflect the situation of "real world" CRT patients, and are therefore important for therapy optimization of these individuals. The study is further limited by the fact that we focused on the necessity and possibilities for CRT optimization, and as such did not assess clinical or echocardiographic outcome in these patients, which was beyond the scope of this study. However, evidence is accumulating as indicated above that empirical AV delay programming cannot generally be recommended [17]. As such, our data do indicate that specialist follow-up of CRT recipients is of crucial importance in order to provide optimal care for these complex patients, which was the primary aim of the current study. 


\section{Conclusions and perspective}

In our "real world" cohort, a substantial proportion of patients presented with suboptimal device settings. Data from previous studies demonstrate that device optimization is associated with improved outcome $[17,18]$. Yet, our data indicate that this opportunity is frequently missed in daily clinical practice, underlining the necessity for expert follow-up to deliver optimal care to this challenging group of heart failure patients in order for them to benefit most of their devices.

Conflict of interest: Jan Steffel: Research support from St. Jude Medical, Biotronik and Sorin; consulting honoraria from Biotronik, Medtronic, St. Jude Medical and Sorin; Helene Rempel: None; Alexander Breitenstein: None; Susann Schmidt: None; Mehdi Namdar: Research and educational grant from Biotronik and St. Jude Medical; Nazmi Krasniqi: Research and educational grants from Medtronic; Johannes Holzmeister: Consulting fees from St. Jude Medical and Biotronik; research grants and speaker honoraria from Biotronik, St. Jude Medical, Medtronic and Boston Scientific; Thomas F. Lüscher: Research grants from Biotronik, Medtronic and St. Jude; Frank Ruschitzka: Research grants and consulting fees from Biotronik, speaker honoraria from Biotronik and Boston Scientific; David Hürlimann: Educational grants from Biotronik, Boston Scientific, Medtronic and St. Jude Medical, speaker honoraria and consulting fees from Biotronik, Medtronic, Sorin and St. Jude Medical.

\section{References}

1. Hunt SA, Abraham WT, Chin MH et al. 2009 focused update incorporated into the acc/aha 2005 guidelines for the diagnosis and management of heart failure in adults: A report of the american college of cardiology foundation/american heart association task force on practice guidelines: Developed in collaboration with the international society for heart and lung transplantation. Circulation, 2009; 119: e391-e479.

2. Bristow MR, Saxon LA, Boehmer J et al. Defibrillation in heart failure. I. Cardiac-resynchronization therapy with or without an implantable defibrillator in advanced chronic heart failure. $\mathrm{N} \mathrm{Engl}$ J Med, 2004; 350: 2140-2150.

3. Chung ES, Leon AR, Tavazzi L et al. Results of the predictors of response to crt (prospect) trial. Circulation, 2008; 117: 2608-2616.

4. Bax JJ, Gorcsan J, 3rd. Echocardiography and noninvasive imaging in cardiac resynchronization therapy: Results of the prospect (predictors of response to cardiac resynchronization therapy) study in perspective. J Am Coll Cardiol, 2009; 53: 1933-1943.

5. Auricchio A, Stellbrink C, Sack S et al. Long-term clinical effect of hemodynamically optimized cardiac resynchronization therapy in patients with heart failure and ventricular conduction delay. J Am Coll Cardiol, 2002; 39: 2026-2033.

6. Hürlimann D, Steffel J, Milosevic G et al. Cardiac resynchronization therapy: "Real world" experience from a swiss tertiary center. Cardiovasc Med (Basel), 2010; 13: 334-341.

7. Bertini M, Delgado V, Bax JJ, Van de Veire NR. Why, how and when do we need to optimize the setting of cardiac resynchronization therapy? Europace, 2009; 11 (suppl. 5): v46-v57.

8. O'Donnell D, Nadurata V, Hamer A, Kertes P, Mohamed U. Long-term variations in optimal programming of cardiac resynchronization therapy devices. Pacing Clin Electrophysiol, 2005; 28 (suppl. 1): S24-S26.

9. Porciani MC, Dondina C, Macioce R et al. Temporal variation in optimal atrioventricular and interventricular delay during cardiac resynchronization therapy. J Card Fail, 2006; 12: 715-719.

10. Sawhney NS, Waggoner AD, Garhwal S, Chawla MK, Osborn J, Faddis MN. Randomized prospective trial of atrioventricular delay programming for cardiac resynchronization therapy. Heart Rhythm, 2004; 1: 562-567.

11. Morales MA, Startari U, Panchetti L, Rossi A, Piacenti M. Atrioventricular delay optimization by Doppler-derived left ventricular $\mathrm{dp} / \mathrm{dt}$ improves 6-month outcome of resynchronized patients. Pacing Clin Electrophysiol, 2006; 29: 564-568.

12. Hardt SE, Yazdi SH, Bauer A et al. Immediate and chronic effects of AV-delay optimization in patients with cardiac resynchronization therapy. Int J Cardiol, 2007; 115: 318-325.

13. Vidal B, Tamborero D, Mont L et al. Electrocardiographic optimization of interventricular delay in cardiac resynchronization therapy: A simple method to optimize the device. J Cardiovasc Electrophysiol, 2007; 18: 1252-1257.

14. Kedia N, Ng K, Apperson-Hansen C et al. Usefulness of atrioventricular delay optimization using Doppler assessment of mitral inflow in patients undergoing cardiac resynchronization therapy. Am J Cardiol, 2006; 98: 780-785.

15. Ellenbogen KA, Gold MR, Meyer TE et al. Primary results from the smartdelay determined av optimization: A comparison to other AV delay methods used in cardiac resynchronization therapy (SMART-AV) trial: A randomized trial comparing empirical, echocardiography-guided, and algorithmic atrioventricular delay programming in cardiac resynchronization therapy. Circulation, 2010; $122:$ 2660-2668.

16. Auricchio A, Stellbrink C, Block M et al. Effect of pacing chamber and atrioventricular delay on acute systolic function of paced patients with congestive heart failure. The pacing therapies for congestive heart failure study group. The guidant congestive heart failure research group. Circulation, 1999; 99: 2993-3001.

17. Brenyo A, Kutyifa V, Moss AJ et al. Atrioventricular delay programming and the benefit of cardiac resynchronization therapy in MADIT-CRT. Heart Rhythm, 2013; 10: 1136-1143.

18. Delnoy PP, Ritter P, Naegele $\mathrm{H}$ et al. Association between frequent cardiac resynchronization therapy optimization and long-term clinical response: A post hoc analysis of the clinical evaluation on advanced resynchronization (CLEAR) pilot study. Europace, 2013; 15: 1174-1181.

19. Cleland JG, Daubert JC, Erdmann E et al. The effect of cardiac resynchronization on morbidity and mortality in heart failure. N Engl J Med, 2005; 352: 1539-1549.

20. Abraham WT, Fisher WG, Smith AL et al. Cardiac resynchronization in chronic heart failure. N Engl J Med, 2002; 346: 1845-1853.

21. Meluzin J, Novak M, Mullerova J et al. A fast and simple echocardiographic method of determination of the optimal atrioventricu- 
lar delay in patients after biventricular stimulation. Pacing Clin Electrophysiol, 2004; 27: 58-64.

22. van Gelder BM, Bracke FA, Meijer A, Pijls NH. The hemodynamic effect of intrinsic conduction during left ventricular pacing as compared to biventricular pacing. J Am Coll Cardiol, 2005; 46: 2305-2310.

23. Pires LA, Abraham WT, Young JB, Johnson KM; MIRACLE Investigators M-I. Clinical predictors and timing of New York Heart Association class improvement with cardiac resynchronization therapy in patients with advanced chronic heart failure: Results from the multicenter InSync randomized clinical evaluation (MIRACLE) and multicenter InSync ICD randomized clinical evaluation (MIRACLE-ICD) trials. Am Heart J, 2006; 151: 837-843.

24. Vatasescu R, Berruezo A, Mont L et al. Midterm 'super-response' to cardiac resynchronization therapy by biventricular pacing with fusion: Insights from electro-anatomical mapping. Europace, 2009; 11: 1675-1682.

25. Butter C, Auricchio A, Stellbrink C et al. Effect of resynchronization therapy stimulation site on the systolic function of heart failure patients. Circulation, 2001; 104: 3026-3029.

26. Sogaard P, Egeblad H, Pedersen AK et al. Sequential versus simultaneous biventricular resynchronization for severe heart failure: Evaluation by tissue Doppler imaging. Circulation, 2002; 106: 2078-2084.

27. Vanderheyden M, De Backer T, Rivero-Ayerza M et al. Tailored echocardiographic interventricular delay programming further optimizes left ventricular performance after cardiac resynchronization therapy. Heart Rhythm, 2005; 2: 1066-1072.

28. Leon AR, Abraham WT, Brozena S et al.; InSync IIICSI. Cardiac resynchronization with sequential biventricular pacing for the treatment of moderate-to-severe heart failure. J Am Coll Cardiol, 2005; 46: 2298-2304

29. Rao RK, Kumar UN, Schafer J, Viloria E, De Lurgio D, Foster E. Reduced ventricular volumes and improved systolic function with cardiac resynchronization therapy: A randomized trial comparing simultaneous biventricular pacing, sequential biventricular pacing, and left ventricular pacing. Circulation, 2007; 115: 2136-2144.

30. Boriani G, Muller CP, Seidl KH et al. Resynchronization for the HemodYnamic Treatment for Heart Failure Management III. Randomized comparison of simultaneous biventricular stimulation versus optimized interventricular delay in cardiac resynchronization therapy. The resynchronization for the hemodynamic treatment for heart failure management II implantable cardioverter defibrillator (RHYTHM II ICD) study. Am Heart J, 2006; 151: 1050-1058.

31. Whinnett ZI, Davies JE, Willson K et al. Haemodynamic effects of changes in atrioventricular and interventricular delay in cardiac resynchronisation therapy show a consistent pattern: Analysis of shape, magnitude and relative importance of atrioventricular and interventricular delay. Heart, 2006; 92: 1628-1634.

32. Corley SD, Epstein AE, DiMarco JP et al.; Investigators A. Relationships between sinus rhythm, treatment, and survival in the atrial fibrillation follow-up investigation of rhythm management (AFFIRM) study. Circulation, 2004; 109: 1509-1513.

33. Gasparini M, Auricchio A, Metra M et al. Multicentre Longitudinal Observational Study G. Long-term survival in patients undergoing cardiac resynchronization therapy: The importance of performing atrio-ventricular junction ablation in patients with permanent atrial fibrillation. Eur Heart J, 2008; 29: 1644-1652. 